\title{
EXTREMAL IRREGULAR DIGRAPHS
}

\author{
JoAnna Górska, ZDZiseaW Skupień \\ AGH Kraków \\ al. Mickiewicza 30, 30-059 Kraków, Poland \\ e-mail: gorska@agh.edu.pl \\ skupien@agh.edu.pl \\ Zyta Dziechcińska-Halamoda, Zofia Majcher
}

AND

JERZY MiCHAEL

Institute of Mathematics and Informatics

Opole University

ul. Oleska 48, 45-052 Opole, Poland

e-mail: zdziech@uni.opole.pl

majcher@math.uni.opole.pl

michael@uni.opole.pl

\begin{abstract}
A digraph is called irregular if its distinct vertices have distinct degree pairs. An irregular digraph is called minimal (maximal) if the removal of any arc (addition of any new arc) results in a non-irregular digraph. It is easily seen that the minimum sizes among irregular $n$-vertex whether digraphs or oriented graphs are the same and are asymptotic to $(\sqrt{2} / 3) n^{3 / 2} ;$ maximum sizes, however, are asymptotic to $n^{2}$ and $n^{2} / 2$, respectively. Let $s$ stand for the sum of initial positive integers, $s=1,3,6, \ldots$ An oriented graph $H_{s}$ and a digraph $F_{s}$, both large (in terms of the size), minimal irregular, and on any such $s$ vertices, $s \geq 21$, are constructed in [Large minimal irregular digraphs, Opuscula Math. 23 (2003) 21-24], co-authored by Z. D-H. and three more of the present co-authors (Z.M., J.M., Z.S.). In the present paper we nearly complete these constructions. Namely, a large minimal irregular digraph $F_{n}$, respectively oriented graph $H_{n}$, are constructed for any of remaining orders $n, n>21$, and of size asymptotic to $n^{2}$, respectively to $n^{2} / 2$. Also a digraph $\Phi_{n}$ and an oriented graph $G_{n}$, both small maximal irregular of any order $n \geq 6$, are constructed. The asymptotic value of the size of $G_{n}$ is at least
\end{abstract}


$(\sqrt{2} / 3) n^{3 / 2}$ and is just the least if $n=s \rightarrow \infty$, but otherwise the value is at most four times larger and is just the largest if $n=s-1 \rightarrow \infty$. On the other hand, the size of $\Phi_{n}$ is of the asymptotic order $\Theta\left(n^{3 / 2}\right)$.

Keywords: irregular digraph, oriented graph, minimal subdigraph, maximal subdigraph, asymptotic size.

2010 Mathematics Subject Classification: 05C07, 05C20, 05C30, 05C35.

\section{REFERENCES}

[1] G. Chartrand and L. Lesniak, Graphs \& Digraphs, 3rd Edition (Chapman \& Hall, 1996).

[2] Z. Dziechcińska-Halamoda, Z. Majcher, J. Michael and Z. Skupień, Large minimal irregular digraphs, Opuscula Math. 23 (2003) 21-24.

[3] Z. Dziechcińska-Halamoda, Z. Majcher, J. Michael and Z. Skupień, Extremum degree sets of irregular oriented graphs and pseudodigraphs, Discuss. Math. Graph Theory 26 (2006) 317-333.

doi:10.7151/dmgt.1323

[4] M. Gargano, J.W. Kennedy and L.V. Quintas, Irregular digraphs, Congr. Numer. 72 (1990) 223-231.

[5] J. Górska, Z. Skupień, Z. Majcher and J. Michael, A smallest irregular oriented graph containing a given diregular one, Discrete Math. 286 (2004) 79-88. doi:10.1016/j.disc.2003.11.049

[6] Z. Majcher, J. Michael, J. Górska and Z. Skupień, The minimum size of fully irregular oriented graphs, Discrete Math. 236 (2001) 263-272. doi:10.1016/S0012-365X(00)00446-5

[7] Z. Skupień, Problems on fully irregular digraphs, in: Z. Skupień and R. Kalinowski, guest eds., Discuss. Math. Graph Theory 19 (1999) 253-255.

doi:10.7151/dmgt.1102

Received 29 June 2016

Revised 7 February 2017

Accepted 7 February 2017 